\title{
The effects of Gaussian beams on optical coherence tomography
}

\author{
Chun-Chu Liu ${ }^{\mathrm{a}}$, Chi-Hsiang Cheng ${ }^{\mathrm{b}}$, Chien-Wei Chiu ${ }^{\mathrm{b}}$ and I-Jen $\mathrm{Hsu}^{\mathrm{b} *}$ \\ ${ }^{a}$ Department of Electrophysics, National Chiao Tung University, Hsinchu, 300, Taiwan, \\ ${ }^{b}$ Department of Physics, Chung Yuan Christian University, Chungli 32023, Taiwan
}

\begin{abstract}
The theory of optical coherence tomography (OCT) was conventionally considered as the light beams propagating in the system to be in the forms of planar waves. However, the actual behaviors of the light beams in an OCT system are more likely to be Gaussian beams. With the consideration of the light beam passing through the focal lens in the sample arm to be a Gaussian beam, we deduced the theory of OCT in an analytic form. We also simulated and analyzed the interference signals with different positions of the photodetector and the interface in the sample as well as their transverse patterns spectrally. The results were demonstrated by experiments with a Fourier-domain OCT system.
\end{abstract}

Keywords: Gaussian beam, optical coherence tomography, interference, ultra-broadband, transverse pattern

\section{INTRODUCTION}

Optical coherence tomography (OCT) is a noninvasive imaging technique to extract cross-sectional information from biological tissues ${ }^{1,2}$. It has been rapidly developed and is useful in biomedical applications ${ }^{1-7}$. Among the alternative configurations of OCT systems, Fourier-domain OCT (FD-OCT) records the information as the function of frequency and obtains the structure of a tissue with Fourier transformation ${ }^{8-11}$. A typical OCT system is based on the configuration of a Michelson interferometer. The theory of OCT was conventionally considered as the light beams propagating in the system to be in the forms of planar waves. Under such an assumption, the theory of OCT is quite simple and is consistent with most of the experimental results. However, the actual behaviors of the light beams in an OCT system are more likely to be Gaussian beams ${ }^{12,13}$.

While considering the effects of Gaussian beams, the interference signals in an OCT system will be different to that resulting from planar waves. Furthermore, the effects will be more crucial when an ultra-broadband light source was used to achieve a high-resolution imaging, which is one of the most important issues in the current development of OCT. The problem of the larger deviation of objective lens from focal plane makes the fail description for the mirror position by the interference signals ${ }^{14}$.

In this work, we develop the mathematical expression for the light beam from the sample arm that has the characteristics of a Gaussian beam. We calculated the interference signals received by the detector, and simulated the effects of Gaussian beam in the interference signals with the sample mirror placed at different positions deviated from the focal plane of the objective. The theoretic predictions were also demonstrated with a FD-OCT system.

\section{THEORY}

A typical OCT system is based on the configuration of a Michelson interferometer as shown in Fig. 1. The reference mirror moves longitudinally $(x)$ and the sample mirror is put at a distance $S$ from the focal plane of the objective lens.

After reflected by the beamsplitter, the light beam propagates to and reflected by the reference mirror and finally stop at the detector with a traveling distance $\ell_{R}$, while the optical length of sample arm is $\ell_{S}$,

$$
\begin{aligned}
& \ell_{R}=2 L_{R}+L_{D}=2(l+f+x)+L_{D}, \\
& \ell_{S}=2 L_{S}+L_{D}=2(l+f+S)+L_{D},
\end{aligned}
$$

Optical Coherence Tomography and Coherence Techniques III, edited by Peter E. Andersen, Zhongping Chen, Proc. of SPIE-OSA Biomedical Optics, SPIE Vol. 6627, 66270T, (C) 2007 SPIE-OSA · 1605-7422/07/\$18 


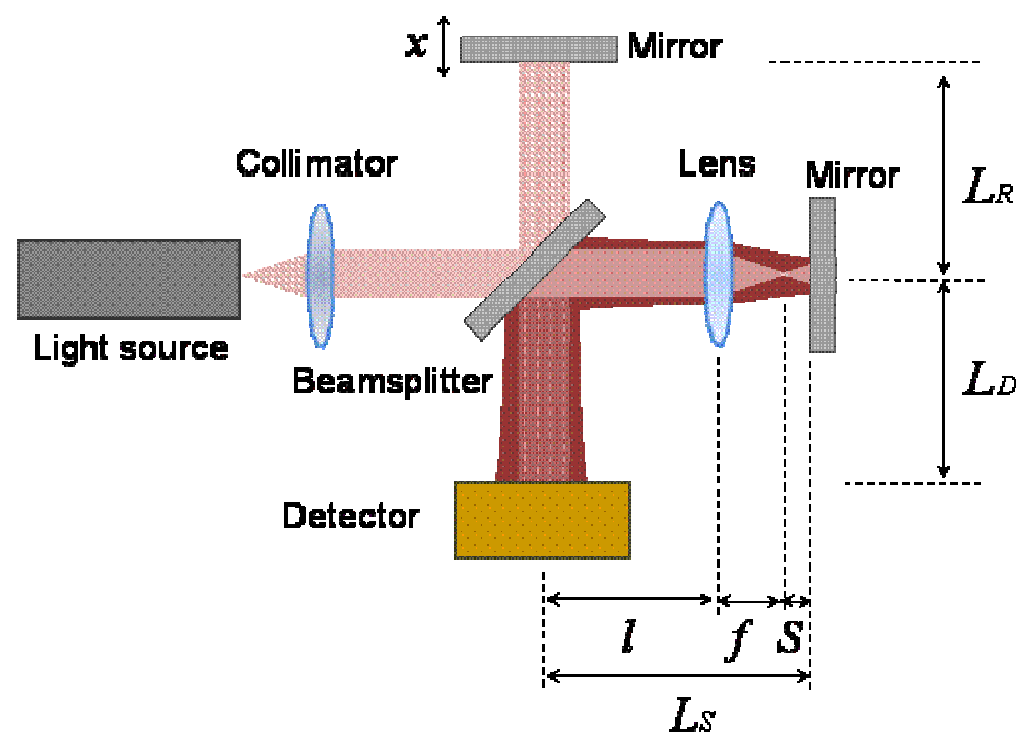

Fig. 1. The OCT system.

where $f$ is the focal length of the objective lens, $l$ is the distance between the beam splitter and focal plane, and $\ell_{D}$ is the distance from beam splitter to the detector. The required time for the sample and reference beams to travel from the beam splitter, reflected by the mirror, and finally arrived the detector are $\ell_{S} / c$ and $\ell_{R} / c$, respectively. We suppose the light beam of the reference arm is the planar wave in the whole journey after the collimator. The wave function of a planar wave is the function of $\omega, z$, and $t$,

$$
E_{R}(\omega, z, t)=A_{0}(\omega) \exp [i(\omega t-k z)],
$$

where $A_{0}(\omega)$ is the spectral amplitude and the experimental measurement is the power spectral density $\left|A_{0}(\omega)\right|^{2}$.

On the other hand, the signal of the sample arm is supposed to be a Gaussian distribution beam since it has been focused by the objective lens. One can obtain the beam waist $W_{0}$ by the incident beam diameter $D$,

$$
W_{0}=\frac{2 \lambda f}{\pi D}=\frac{4 c f}{\omega D},
$$

as shown in Fig.2 Rayleigh range

$$
z_{0}=\frac{\pi W_{0}^{2}}{\lambda}=\frac{\omega W_{0}^{2}}{2 c} .
$$

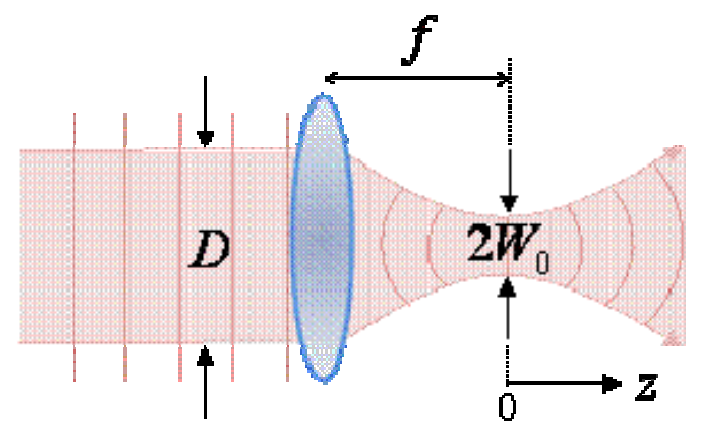

Fig. 2. The sample single propagates through an objective lens and focus with waist $W_{0}$ at where $z=0$. 
While the Gaussian beam at a distance from the waist is smaller than $z_{0}$, the radius of the curvature of the wavefront is approximate to the planar wave, i.e. at waist the radius is infinite. After exceeding the Rayleigh range, the radius will gradually increase.

We set the traveling coordinate $z$ for the sample beam at the waist as the beginning position $z=0$, as shown in Fig.2, the light beam is reflected by the mirror, goes back passing the lens and splitter, and finally arrives the detector. To estimate the beam size and radius of the curvature of wavefront, we utilize the ABCD matrix,

$$
\left(\begin{array}{ll}
A & B \\
C & D
\end{array}\right)=\left(\begin{array}{ll}
1 & L \\
0 & 1
\end{array}\right)\left(\begin{array}{cc}
1 & 0 \\
-\frac{1}{f} & 1
\end{array}\right)\left(\begin{array}{cc}
1 & f+2 S \\
0 & 1
\end{array}\right)=\left(\begin{array}{cc}
1-\frac{L}{f} & f+2 S-\frac{2 L S}{f} \\
-\frac{1}{f} & -\frac{2 S}{f}
\end{array}\right),
$$

with $L=l+f$. We can obtain the beam size,

$$
W_{D}=\left(\frac{2 \pi c}{\omega} \times \frac{\left(f^{2}+2 f S-2 L S\right)^{2}+(L-f)^{2} \cdot z_{0}^{2}}{z_{0} f \pi}\right)^{1 / 2},
$$

and the radius of curvature of the wavefront of Gaussian beam,

$$
R_{D}=\frac{\left(f^{2}+2 f S-2 L S\right)^{2}+(L-f)^{2} \cdot z_{0}^{2}}{2 S\left(2 L S-2 f S-f^{2}\right)+(L-f) \cdot z_{0}^{2}},
$$

at detector as shown in Fig. 3. At detector center the traveling coordinate of the sample signal is $z_{1}$

$$
z_{1}=2 S+f+l+L_{D}
$$

and deviated from the center at transverse coordinate $\rho_{i}$, the traveling coordinate is $z_{i}$,

$$
z_{i}=R_{i}-\sqrt{R_{i}^{2}-\rho_{i}^{2}}+z_{1}
$$

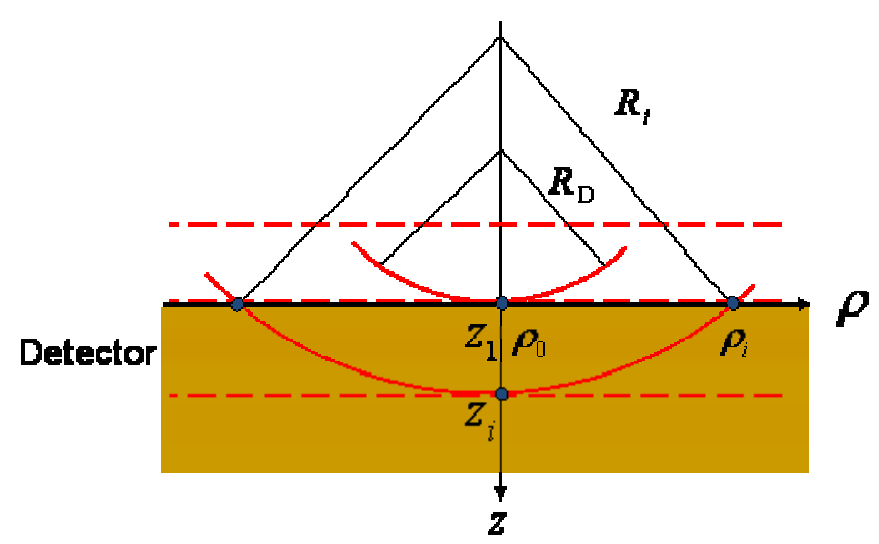

Fig. 3. The sample single propagates through an objective lens and focus with waist $W_{0}$ at where $\mathrm{z}=0$.

Here, we use the cylindrical coordinate, since the Gaussian beam has the cylindrical symmetry at the traversed planar. The wave function of a Gaussian laser beam wave function toward $+\hat{z}$ can be express as the function of $\rho, \omega, z$, and $t$,

$$
E_{S}(\rho, z, \omega, t)=A_{0}(\omega) \frac{W_{0}}{W(z)} \exp \left[\frac{2 \rho^{2}}{W(z)^{2}}\right] \exp \left[-i \frac{\omega}{c} \frac{\rho^{2}}{2 R(z)}\right] \exp [i(\omega t-k z)]
$$

The intensity of the interference between two electric fields in Eqs. (3) and (11) is

$$
I_{D}=\left|\left\langle E_{R}\right\rangle\right|^{2}+\left|\left\langle E_{S}\right\rangle\right|^{2}+\left|\left\langle 2 E_{R} E_{S}^{*}\right\rangle\right|,
$$


with

$$
\begin{gathered}
\left|\left\langle E_{R}\right\rangle\right|^{2}=\left|A_{0}(\omega)\right|^{2}, \\
\left|\left\langle E_{S}\right\rangle\right|^{2}=\left|A_{0}(\omega)\right|^{2}\left(\frac{W_{0}}{W(z)}\right)^{2} \exp \left[\frac{2 \rho^{2}}{W(z)^{2}}\right],
\end{gathered}
$$

and

$$
\begin{aligned}
\left|\left\langle 2 E_{R} E_{S}^{*}\right\rangle\right|=\iint 2\left|A_{0}(\omega)\right|^{2} \frac{W_{0}}{W(z)} \exp \left[\frac{2 \rho^{2}}{W(z)^{2}}\right] \exp \left[-i \frac{\omega}{\mathrm{c}} \frac{\rho^{2}}{2 R(z)}\right] \\
\times \exp \left[i \frac{\omega}{c}\left(\left(\ell_{S}-\ell_{R}\right)-\left(z_{i}-z_{1}\right)\right)\right] 2 \pi \rho d \omega d \rho,
\end{aligned}
$$

in which we integrate with all of the frequency and the beam area over the detector. The parts of $\left|\left\langle E_{R}\right\rangle\right|^{2}$ and $\left|\left\langle E_{S}\right\rangle\right|^{2}$ are dc signals and we only need to consider their cross-interference term $\left|\left\langle 2 E_{R} E_{S}^{*}\right\rangle\right|$ in Eq. (14). Here, the transverse distribution of the interference signal, i.e. the intensity in $\rho$ direction since the signals have the cylindrical symmetry, is

$$
\begin{aligned}
&\left|\left\langle 2 E_{R} E_{S}^{*}\right\rangle\right|_{\rho}=\int 2\left|A_{0}(\omega)\right|^{2} \frac{W_{0}}{W(z)} \exp \left[\frac{2 \rho^{2}}{W(z)^{2}}\right] \exp \left[-i \frac{\omega}{\mathrm{c}} \frac{\rho^{2}}{2 R(z)}\right] \\
& \times \exp \left[i \frac{\omega}{c}\left(\left(\ell_{S}-\ell_{R}\right)-\left(z_{i}-z_{1}\right)\right)\right] d \omega .
\end{aligned}
$$

\section{NUMERICAL AND EXPERIMENTAL RESULTS}

For comparison, we setup a Fourier Domain (FD) OCT system as Fig.1. The light source in our system is a superluminescent diode (SLD D890, Superlum Diodes Ltd.) with a spectral bandwidth of $150 \mathrm{~nm}$ (FWHM) centered at $890 \mathrm{~nm}$ as Fig. 4 and axial resolution of $3.7 \mu \mathrm{m}$ in air. The collimated light (by an object lens: 60X, NA $=0.85, f=$ $2.9 \mathrm{~mm})$ is split half $(50 \%)$ to the reference mirror and half to the reference mirror. At the sample side, a near IR achromatic doublet lens (Thorlabs, Inc.) with focal length $7.5 \mathrm{~mm}$ makes the photon beam reflected by the sample has the character of Gaussian beam; while at the reference side, the photon beam is reflected by the mirror directly and remain to be the planar waves. Finally the interference signals are received by a spectrometer (USB2000, Ocean Optics, Inc.). The data obtained by the spectrometer are the interference intensity in frequency domain. For the time domain distribution one will need to do the Fourier transformation that we do this analysis by software LabVIEW.

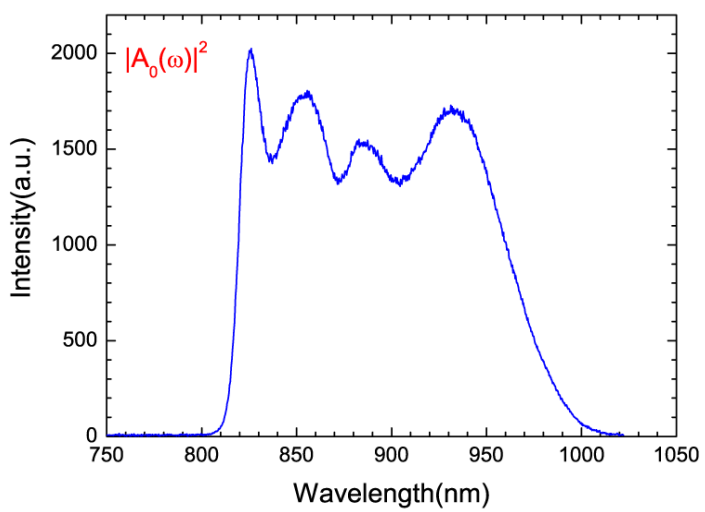

Fig. 4. The spectral density distribution with the center wave length $890 \mathrm{~nm}$ and band width $150 \mathrm{~nm}$. 
The experimental parameters such as spectral intensity $\left|A_{0}(\omega)\right|^{2}$ in Fig. 4 will be used as the input in Eq. (15), as well as $D=0.96 \mathrm{~mm}, L_{D}=32 \mathrm{~cm}, L_{R}=12 \mathrm{~cm}, l=11.25 \mathrm{~cm}, f=7.5 \mathrm{~mm}$, and $L_{S}=l+f+S$.

\subsection{Interference signals}

\subsubsection{Numerical Results}

In Eq. (14), we have calculated the interference intensity including the effect of Gaussian beam. With the same spectral density distribution as in experiment in Fig. 4, we obtain numerical simulation results as shown in Fig. 5. Basically, if the sample mirror is set at the focal plane of the lens $(S=0)$, the incident light will be reflected along the same path of incidence and arrive the detector. The signal pattern is symmetry with respect to the position of $S=0$. However, if $S$ is unequal to zero, the optical path will be totally different. For example, when the sample mirror is put at where $S$ is larger than zero, as shown in Fig.1, the optical path will be reflected outward and the beam size will be enlarged. The interference signal will become asymmetry as $|S|>0$. In our numerical results, the signals have apparently asymmetric as $|S|>150 \mu \mathrm{m}$. We also find that the more asymmetric happens at $S<0$ side. We show the interference signals with $S$ $=0,30,90,150,210,270$, and $330 \mu \mathrm{m}$ in Fig. 5(b) and $S=0,-30,-90,-150,-210,-270$, and $-330 \mu \mathrm{m}$ in Fig. 5(c), respectively.

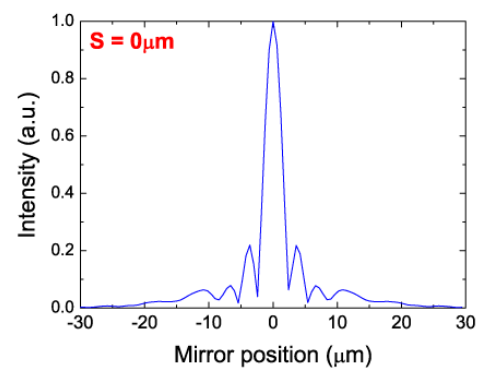

Fig. 5(a). Numerical simulation of the interference signal with $S=0 \mu \mathrm{m}$.
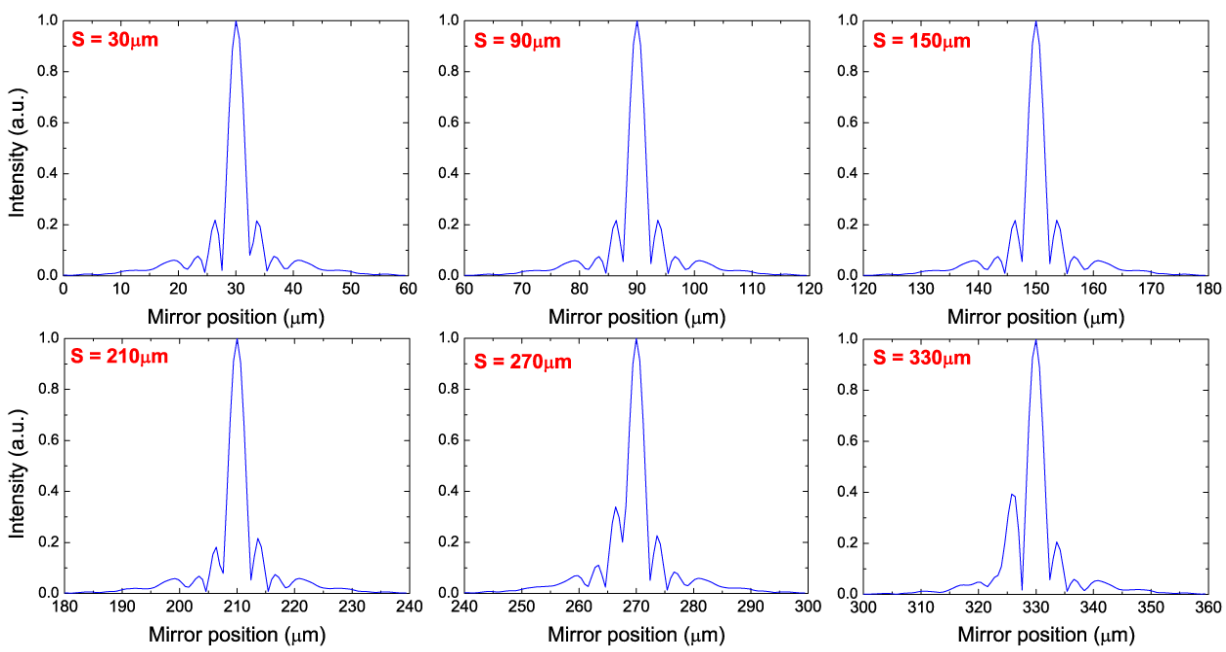

Fig. 5(b). Numerical simulation of the interference signals with $S=30,90,150,210,270$, and $330 \mu \mathrm{m}$. 

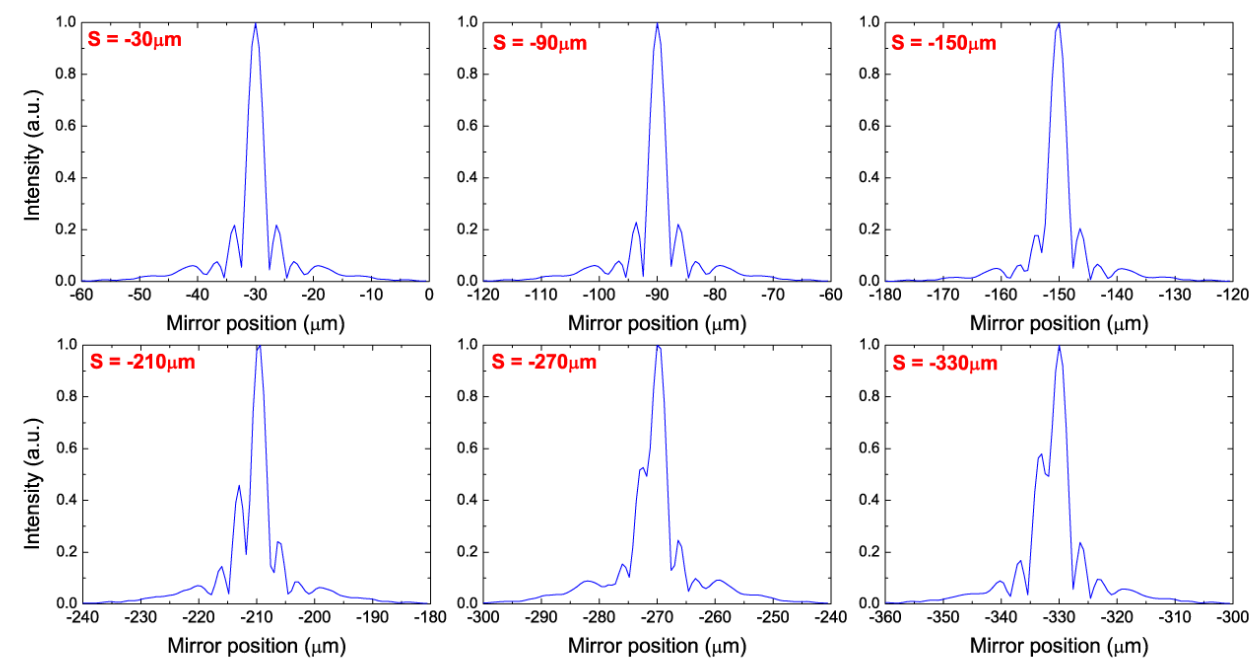

Fig. 5(c). Numerical simulation of the interference signals with $S=-30,-90,-150,-210,-270$, and $-330 \mu \mathrm{m}$.

\subsubsection{Experimental Results}

In comparison with the theoretic simulation, we list the corresponding experimental results in Fig. 6 which have been analysed with Fast Fourier transformation from the original experimental data that are the frequency domain signals. Except the pattern with $S=0$ with well right and left symmetric, the phenomenon of asymmetries happens in other patterns. As the distance $S$ enlarges, in Figs. 6(b) and 6(c), the signal at left side enhanced. In which the abnormal behavior of patterns $S= \pm 30 \mu \mathrm{m}$ is happened by the self-interference effect at $S=0$. All of the patterns except $S=0$ have the phenomenon of asymmetries in their signals in experimental results. The theoretical and experimental results are qualitatively consistent. Both results can be found the higher asymmetric effect in $S<0$, Figs. 5(c) and 6(c), than in $S>0$, Figs. 5(b) and 6(b).

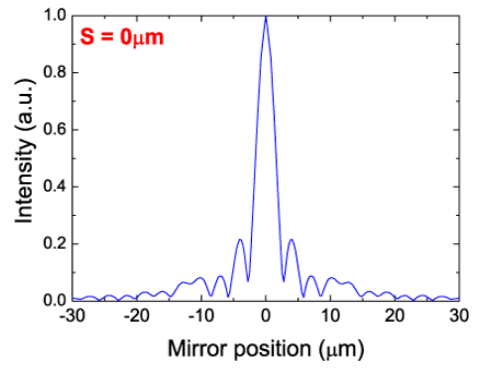

Fig. 6(a). Experimental results of the interference signal with $S=0 \mu \mathrm{m}$. 

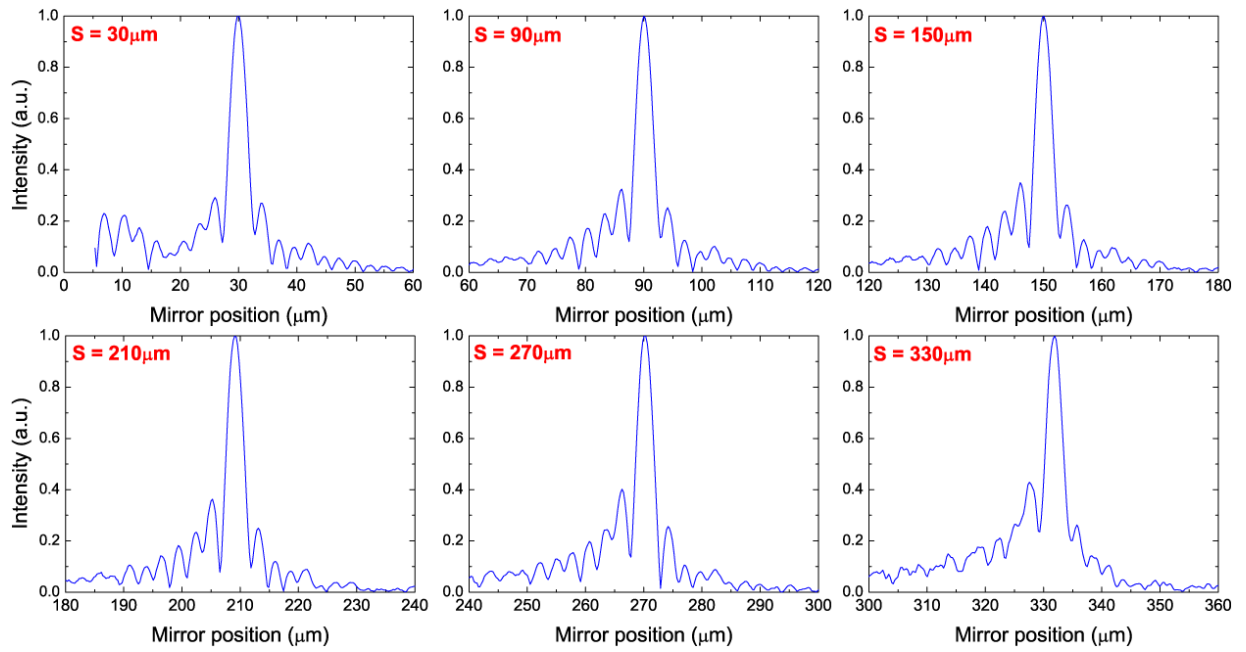

Fig. 6(b). Experimental results of the interference signals with $S=30,90,150,210,270$, and $330 \mu \mathrm{m}$.
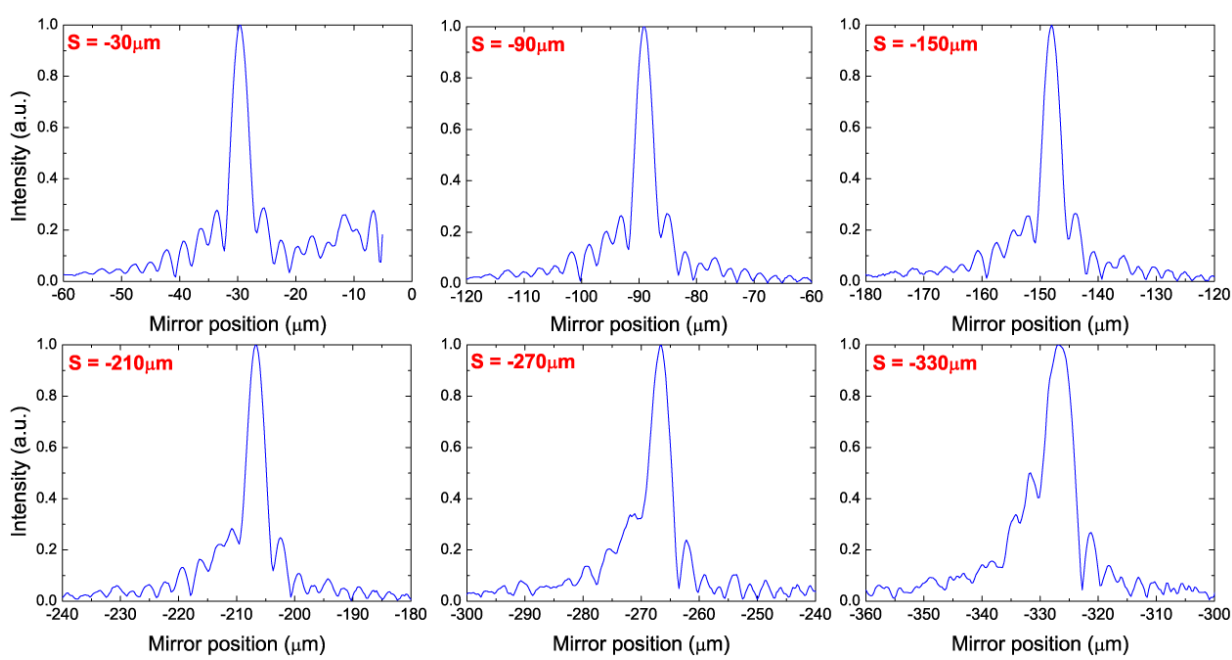

Fig. 6(c). Experimental results of the interference signals with $S=-30,-90,-150,-210,-270$, and $-330 \mu \mathrm{m}$.

\subsection{Transverse distributions ( $\rho$ direction distributions)}

With Eq. (15), we also simulate the transverse distribution the interferences of the reference and sample beams with sample mirror set in different positions. Figure 7 shows the numerical simulations results with $S=0, \pm 30, \pm 60, \pm 90$, $\pm 150, \pm 210, \pm 270$, and $\pm 330 \mu \mathrm{m}$. Observing Fig. 7(b) with $S>0$ and Fig. 7(c) with $S<0$, we can conclude that theory calculate reveal that the sample beam at the detector will enlarge its beam size with $|S|$ growing. The later one, $S<0$, spreads more conspicuously as $S$ grows. 


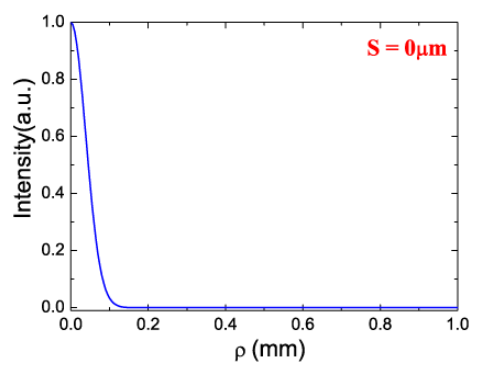

Fig. 7(a). Numerical simulation of transverse interference signals with $S=0 \mu \mathrm{m}$.
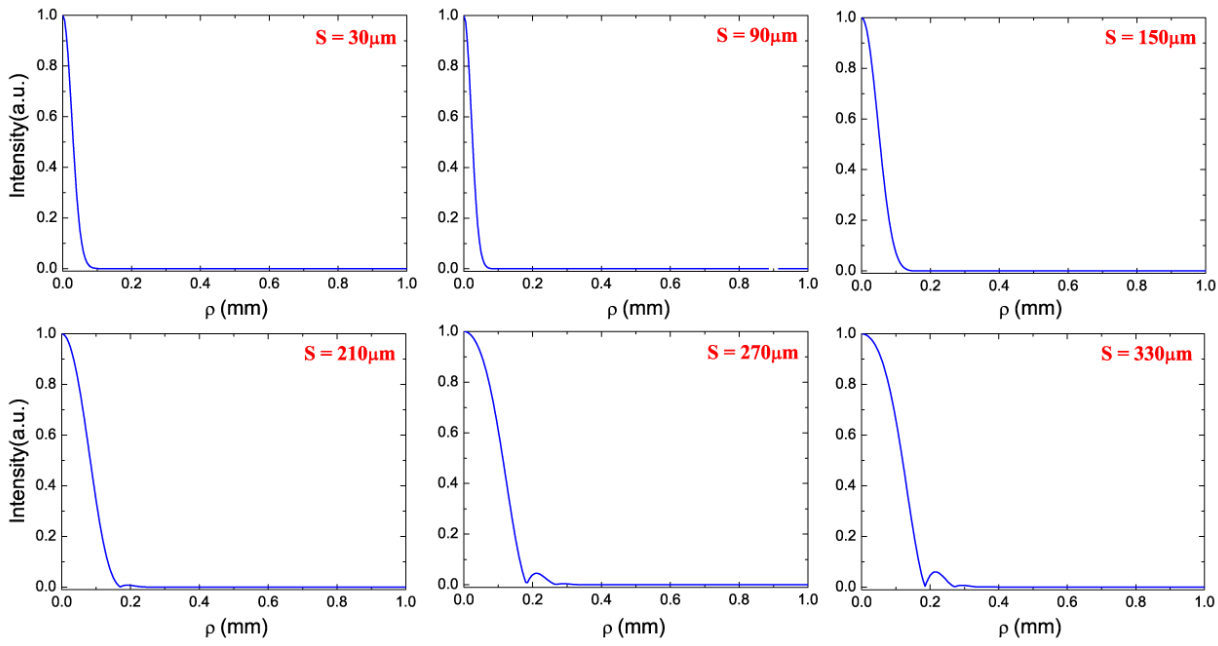

Fig. 7(b). Numerical simulation of transverse interference signals with $S=30,90,150,210,270$, and $330 \mu \mathrm{m}$.
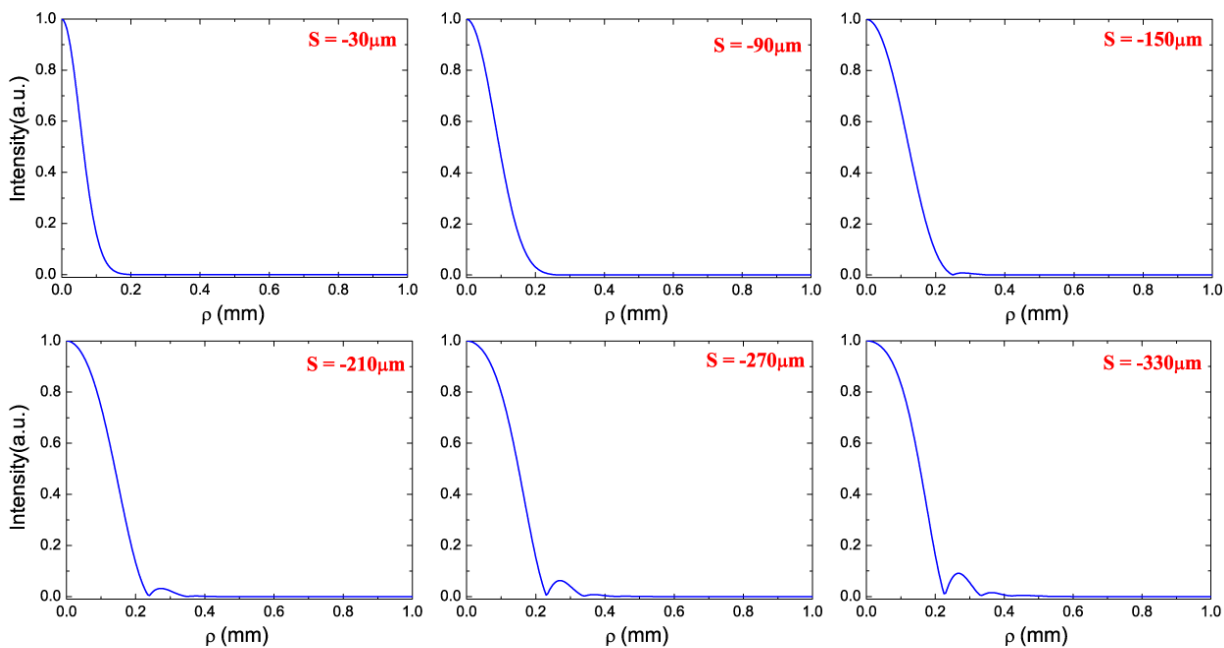

Fig. 7(c). Numerical simulation of transverse interference signals with $S=-30,-90,-150,-210,-270$, and $-330 \mu \mathrm{m}$. 


\section{CONCLUSIONS}

We have developed the mathematical expression for the light beam from the sample arm that has the un-neglected effects of Gaussian beam. We have calculated the interference intensity between reference and sample signals at the detector. We test the effects of Gaussian beam in the interference signals with the sample mirror is put at different positions from the focal plane of the objective lens. We have set up a Fourier domain (FD) OCT systems and succeeded to demonstrate our theoretic predictions that the interference signals are affected by the characters of the Gaussian distribution waves. We also have studied the transverse distributions for different sample positions by theory simulations and obtained the more spreading signals in cross-sections as sample mirror moving away the focal plane of the objective lens.

\section{ACKNOWLEDGEMENTS}

This research was supported in part by the National Science Council of Republic of China under the Grant No. NSC 952112-M-033-004 and NSC-95-2112-M-009-041-MY2.

\section{REFERENCES}

1. D. Huang, E. A. Swanson, C. P. Lin, J. S. Schuman, W. G. Stinson, W. Chang, M. R. Hee, T. Flotte, K. Gregory,C. A. Puliafito, and J. G. Fujimoto, "Optical coherence tomography," Science 254, 1178-1181 (1991).

2. E.g., James G. Fujimoto, "Handbook of optical coherence tomography," Chapter 1, Edited by B.E. Bouma, G.J.Tearney, Marcel Dekker, Inc. (2002).

3. E. A. Swanson, D. Huang, M. R. Hee, J. G. Fujimoto, C. P. Lin, and C. A. Puliafito, "High-speed optical coherence domain reflectometry," Opt. Lett. 17, 151-153(1992).

4. A. F. Fercher, W. Drexler, C. K. Hitzenberger, and T. Lasser, "Optical coherence tomography - principles and applications," Rep. Prog. Phys. 66, 239-303 (2003).

5. W. Drexler, U. Morgner, F. X. Kartner, C. Pitris, S. A. Boppart, X. D. Li, E. P. Ippen, and J. G. Fujimoto, "In vivo ultrahigh-resolution optical coherence tomography," Opt. Lett. 24, 1221-1223 (1999).

6. H. Lim, Y. Jiang, Y. M. Wang, Y. C. Huang, Z. P. Chen, and F. W. Wise, "Ultrahigh-resolution optical coherence tomography with a fiber laser source at $1 \mu \mathrm{m}$," Opt. Lett. 30, 1171-1173 (2005).

7. B. Povazay, K. Bizheva, A. Unterhuber, B. Hermann, H. Sattmann, A. F. Fercher, W. Drexler, A. Apolonski, W. J. Wadsworth, J. C. Knight, P. St. J. Russell, M. Vetterlein, and E. Scherzer, "Submicrometer axial resolution optical coherence tomography," Opt. Lett. 27, 1800-1802 (2002).

8. M. Wojtkowski, A. Kowalczyk, R. Leitgeb, and A. F. Fercher, "Full range complex spectral optical coherence tomography technique in eye imaging, "Opt. Lett. 27, 1415-1417 (2002).

9. R. Leitgeb, W. Drexler, A. Unterhuber, B. Hermann, T. Bajraszewski, T. Le, A. Stingl, and A. Fercher, “ Ultrahigh resolution Fourier domain optical coherence tomography," Opt. Express 12, 2156-2165 (2004).

10. R. Leitgeb, C. K. Hitzenberger, and A. F. Fercher, "Performance of Fourier domain vs. time domain optical coherence tomography," Opt. Express 11, 889-894 (2003).

11. Maciej Wojtkowski, Vivek Srinivasan, Tony Ko, James Fujimoto, Andrzej Kowalczyk, and Jay Duker, "Ultrahighresolution, high-speed, Fourier domain optical coherence tomography and methods for dispersion compensation," Opt. Express 12, 2404-2422 (2004).

12. Javier Alda , "Laser and Gaussian Beam Propagation and Transformation," Encyclopedia of Optical Engineering, Marcel Dekker, Inc. (2003).

13. B.E.A. Saleh, and M.C. Teich, "Fundamentals of photonics", Wiley, p80 (1991).

14. Y. Yasuno, J. I. Sugisaka, Y. Sando, Y. Nakamura, S. Makita, M. Itoh, and T. Yatagai, "Non-iterative numerical method for laterally superresolving Fourier domain optical coherence tomography," Opt. Express 14, 1006-1020 (2006). 